\title{
Gliosis-specific transcription factor OASIS coincides with proteoglycan core protein genes in the glial scar and inhibits neurite outgrowth
}

\author{
Ken Iseki ${ }^{1,2}$, Seita Hagino ${ }^{3}$, Takuya Nikaido ${ }^{3}$, Yuxiang Zhang ${ }^{3}$, Tetsuji Mori ${ }^{3}$, Sachihiko YokoYa ${ }^{3}$, Yasukazu \\ Hozumi $^{2}$, Kaoru Goto ${ }^{2}$, Akio Wanaka ${ }^{4}$, and Choichiro Tase ${ }^{5}$ \\ ${ }^{1}$ Department of Emergency and Critical Care Medicine, ${ }^{2}$ Department of Anatomy and Cell Biology, Yamagata University School of \\ Medicine, Iida-nishi 2-2-2, Yamagata 990-9585, Japan; ${ }^{3}$ Department of Cell Science, Institute of Biomedical Sciences, ${ }^{5}$ Department of \\ Emergency and Critical Care Medicine, Fukushima Medical University School of Medicine, 1 Hikarigaoka, Fukushima 960-1295, \\ Japan; and ${ }^{4}$ Department of Anatomy, Nara Medical University School of Medicine, 840 Shijo-cho, Kashihara 634-8521, Japan
}

(Received 9 September 2012; and accepted 10 October 2012)

\begin{abstract}
OASIS gene, a member of the CREB/ATF transcription factor family, is upregulated in gliosis after CNS injury. However it remains to be determined how OASIS is implicated in gliotic reaction. In a glial scar, chondroitin sulfate proteoglycans (CSPGs) are also upregulated, which engenders the inhibition of axonal regeneration. We investigated the functional role of OASIS in gliosis in relation to CSPG core proteins that render lesions non-permissive for regenerating axons. We first examined the gene expression localization of OASIS using several markers in a cryo-injured mouse brain and compared the expression pattern of CSPG core protein genes with that of OASIS in a glial scar by double-labeling in situ hybridization. Our findings suggest that OASIS is induced in proximal reactive astrocytes that exhibit upregulated expression for CSPGs, including NG2 proteoglycan, versican, brevican, neurocan, and phosphacan core. Furthermore, the membrane fraction derived from OASIS-transfected C6 cells inhibits neurite outgrowth of NG108-15 cells, whereas its neurite outgrowth inhibitory effect is abrogated after chondroitinase ABC treatment. OASIS is likely to be involved in the regulatory mechanism of non-permissive environments for axonal outgrowth.
\end{abstract}

Responses of glial cells to central nervous system (CNS) injury cause the formation of a glial scar (gliosis). Reactive astrocytes, characterized by the intense expression of glial fibrillary acidic protein (GFAP), are the main component of the scar $(8,9$, 39). Among the cells in the lesion, reactive astrocytes are known to express inhibitory substances for axonal growth, such as chondroitin sulfate proteoglycans (CSPGs). Thereby, they become a major impediment to the successful restoration of brain

Address correspondence to: Ken Iseki, M.D. Ph.D. Department of Emergency and Critical Care Medicine, Yamagata University School of Medicine, Iida-nishi 2-2-2, Yamagata 990-9585, Japan

Tel: +81-23-628-5422, Fax: +81-23-628-5423

E-mail: ken@med.id.yamagata-u.ac.jp function (11), as exemplified by the fact that growing axons of transplanted neurons stop at and turn away from regions that are rich in chondroitin sulfate moiety in spinal cord injury (6).

To investigate the mechanisms regulating such glial responses, we performed differential display screening of genes that were induced specifically in long-term cultured astrocytes, a potential in vitro gliosis model (24), and identified a gene named old astrocyte specifically induced substance (OASIS). OASIS, which encodes a transcription factor belonging to the CREB/ATF family (20), is expressed in the cortex, hippocampus and thalamus of the adult brain under normal conditions. In terms of the gene expression, OASIS mRNA decreases in intensity during postnatal period, although it is upregulated in the brain lesion in a distribution pattern resembling 
that of GFAP-positive astrocytes (20,30).

In addition, previous reports have described that OASIS mRNA is expressed in osteoblasts and preosteoblasts (35), each of which actively secretes extracellular matrices such as CSPGs. In this regard, gliotic tissue also expresses CSPGs, including the NG2 proteoglycan $(29,31)$, versican $(2,38,40)$, brevican (28), phosphacan (33), and neurocan (17, 33). These CSPGs are shown to inhibit nerve regeneration $(12,41,42,46)$. From these findings, we hypothesized that OASIS might participate in transcription of some, if not all, of the CSPG core proteins that are upregulated in the glial scar.

In this study, we first examined the cell types expressing OASIS mRNA by double labeling with various cell markers and compared details of the distribution pattern of OASIS mRNA with those of CSPG core proteins. Here we show in a cryo-injured brain that OASIS is expressed in proximal reactive astrocytes that exhibit upregulated expression for CSPGs. Furthermore, we show that a membrane fraction derived from OASIS-transfected C6 cells significantly inhibits neurite outgrowth of NG108-15 cells, suggesting that OASIS contributes to the nonpermissive environment for axonal outgrowth, presumably through regulation of the proteoglycan expression in reactive astrocytes.

\section{MATERIALS AND METHODS}

Animals and tissue preparation. Our experiments used 30 adult male ICR mice. All experimental procedures were approved by the institutional committee of Fukushima Medical University. The following surgical procedures were performed under the combined anesthesia of diethyl ether inhalation and pentobarbital injection. Cryo-injury was inflicted by placing a lead probe that had been pre-chilled with liquid nitrogen on the cranium for $1 \mathrm{~min}$. The mice were perfused transcardially with phosphate-buffered saline (PBS) followed by $4 \%$ paraformaldehyde (PFA) under deep ether anesthesia at 2, 4, 7, and 14 days after cryo-injury. The brains were dissected, post-fixed by immersion in $4 \% \mathrm{PFA}$ at $4^{\circ} \mathrm{C}$ for $24 \mathrm{~h}$, and equilibrated in 30\% sucrose in diethyl pyrocarbonate-treated PBS. They were embedded in OCT compound (Tissue Tek; Sakura Finetek, Inc., Torrance, CA, USA) with powdered dry ice and stored at $-80^{\circ} \mathrm{C}$ until use. Coronal sections $(10 \mu \mathrm{m}$ thick $)$ were cut on a cryostat and mounted onto silanecoated slides. Sections were then subjected to in situ hybridization or immunohistochemical analyses.
Probes and in situ hybridization. In situ hybridization using a digoxigenin-labeled cRNA probe was performed as described previously $(20,35)$. Digoxigenin-labeled cRNA probes (antisense and sense probes) were synthesized using T7 or SP6 RNA polymerase (Promega Corp., Madison, WI, USA) in the presence of digoxigenin-labeled UTP by in vitro transcription using OASIS and CSPG core protein cDNAs. OASIS cDNA fragment of 920 bp (nucleotides 1090-2010) (20) was used, which excluded the basic domain and leucine zipper sequences to avoid possible cross-hybridization with known family members (35). The CSPG core protein cDNAs were obtained from the IMAGE consortium cDNA library. The neurocan cDNA was kindly provided by Dr. Y. Tokita (Aichi Human Service Center). Genbank accession numbers for the cDNAs used were the following: AI019805 for NG2 proteoglycan, AI323043 for versican, AA386748 for phosphacan, AU080821 for brevican, and M97161 for neurocan. We confirmed the validity of the cDNAs by sequencing and by in situ hybridization on mouse embryo sections to ensure no cross-reaction between probes. For single in situ hybridization detection of digoxigenin-labeled cRNA probe, anti-digoxigenin antibody conjugated to alkaline phosphatase (Boehringer-Mannheim) was applied at a dilution of $1: 500$, and color was developed with nitroblue tetrazolium/5-bromo-4-chloro-indolyl phosphate (NBT/BCIP) according to manufacturer's protocol.

In situ hybridization combined with immunohistochemistry. In situ hybridization combined with immunohistochemistry was performed as described previously with a small modification (22). In situ hybridization was performed using OASIS probe covalently linked to digoxigenin, as visualized with a rhodamine-conjugated anti-digoxigenin antibody (1 : 100 dilution; F. Hoffmann-La Roche Ltd., Indianapolis, IN, USA). After blocking in 5\% skim milk in PBS for $30 \mathrm{~min}$, sections were incubated with one of the following antibodies: rabbit anti-GFAP antibody (Biomeda, Foster City, CA, USA), hamster anti-target of the anti-proliferative (anti-TAPA) antibody (a marker for proximal reactive astrocytes, 1: 200 dilution; BD Bioscience PharMingen, San Diego, CA, USA) $(7,15,37)$, mouse monoclonal antibody MAB328 (a marker for mature oligodendrocytes, $1: 200$ dilution; Chemicon International Inc., Temecula, CA, USA), or rabbit anti-ionized calcium binding adaptor molecule-1 (anti-Iba1) antibody (a specific marker for macrophage/microglia, $2 \mu \mathrm{g} / \mathrm{mL}$, kindly provided by Dr. S. Kohsaka, Na- 
tional Institute of Neuroscience) (7, 16, 21, 25, 26). Following three washes with PBS, the GFAP and Iba1 immunoreactivities were detected using an FITC-conjugated anti-rabbit IgG (1: 40 dilution; American Qualex Antibodies Inc., San Clemente, CA, USA). TAPA-immunoreactivity was detected with a biotinylated goat anti-hamster IgG secondary antibody (1 : 1000 dilution; American Qualex Scientific Products, Cedarlane, Ontario, Canada) followed by a FITC-conjugated streptavidin (1:50 dilution; Cosmobio Co. Ltd., Tokyo, Japan). MAB328-immunoreactivity was visualized with a biotinylated anti-mouse IgG + IgM secondary antibody (KPL; Kirkegaard \& Perry Laboratories, Inc., Gaithersburg, MD, USA) and FITC-conjugated streptavidin using a VECTOR M.O.M. immunodetection kit (Vector Laboratories Inc., Burlingame, CA, USA). All sections were subsequently observed and photographed using a fluorescence laser microscope (Olympus Fluoview FV300; Olympus Corp., Tokyo, Japan) equipped with filters for the simultaneous examination of FITC and rhodamine fluorescence. No crossreaction was observed between secondary and tertiary antibodies (e.g., biotinylated goat anti-hamster IgG antibody did not react with rabbit antiGFAP or Iba1 antibodies).

Double labeling in situ hybridization. Double labeling in situ hybridization was performed as described previously (22). Sections were hybridized with digoxigenin-labeled cRNA probes for CSPG core protein genes and FITC-labeled OASIS cRNA probes. The CSPG core protein gene mRNAs were visualized using a rhodamine-conjugated anti-digoxigenin antibody (1:100 dilution; Hoffmann-La Roche Inc.). OASIS mRNA was detected using an antiFITC antibody conjugated to horseradish peroxidase (1: 500 dilution; Hoffmann-LaRoche Inc.), which was amplified using FITC-conjugated tyramide (TSA Fluorescence System; NEN Life Science Products, Boston, MA, USA).

Cell culture and transfection. The C6 glioma cells were maintained in DMEM containing $10 \%$ fetal calf serum, $10 \mathrm{mM}$ L-glutamine and penicillin $(100 \mathrm{IU} / \mathrm{mL}) /$ streptomycin $(100 \mu \mathrm{g} / \mathrm{mL})$ at $37^{\circ} \mathrm{C}$ and $5 \% \mathrm{CO}_{2}$ in an incubator. Transient or stable expression of a full length of the OASIS cDNA was achieved in C6 cells by transfection with Effectene (QIAGEN Inc., Germantown, MD, USA) according to the manufacturer's instructions. Subsequent selection was performed with G418 $(600 \mu \mathrm{g} / \mathrm{mL}$, Geneticin; Invitrogen Corp., Carlsbad, CA, USA) for stable expression. Control cells were transfected with an expression vector alone in the manner described above.

Neurite outgrowth assay. Two stable clones (OASIS1 and OASIS2) were established from C6 cells transfected with a full-length OASIS cDNA in pCAGGS vector. As controls, we used C6 cells that had stably transfected with a vector alone. Cells were washed three times with ice-cold PBS and collected in icecold PBS with PMSF using a cell scraper. After homogenization, a crude sample was centrifuged at $600 \times g$ for $10 \mathrm{~min}$ to remove cell debris; it was then re-centrifuged at $8000 \times g$ for 10 min to yield a membrane fraction (supernatant). The membrane fraction was coated onto tissue culture dishes and dried up. Then NG108-15 cells (the neuroblastoma $\times$ glioma hybrid cell line) were cultured in the presence of $10 \mathrm{mM}$ dibutyryl cAMP (Sigma, St. Louis, MO, USA) to induce neurite outgrowth on those dishes. Some membrane fractions were treated with chondroitinase ABC (0.02 U/mL; Seikagaku Corp., Tokyo, Japan) for $1 \mathrm{~h}$ to remove chondroitin sulfate moieties before coating according to the previous report (1). After 7 days, NG108-15 cells with neurites were selected randomly $(n>200)$; they were photographed by a blinded observer. The lengths of the neurites on the pictures were measured by another blinded observer. Then the data were summed up to obtain the total neurite length per single cell (mean $\pm \mathrm{SD}$ ). Data for the groups, including vectortransfected cells and OASIS-transfected cells (OASIS1 and OASIS2), were subjected to statistical analyses using ANOVA and Scheffé's test for post hoc multiple comparisons.

\section{RESULTS}

OASIS mRNA expression in proximal reactive astrocytes

On day 7 after cryo-injury, a necrotic lesion was observed in the cerebral cortex. In most cases, the lesion width was approximately $800-1200 \mu \mathrm{m}$. The necrotic lesion was surrounded by densely packed cells, showing reactive gliosis. Expression of OASIS mRNA was increased considerably in the region surrounding the necrotic tissue at this stage (Fig. 1A), which is consistent with a previous report (20). No remarkable signal was detected in adjacent sections hybridized with the sense probe (Fig. 1B).

To determine which cells are responsible for the expression of OASIS mRNA in cryo-injured brain, we performed in situ hybridization in conjunction 


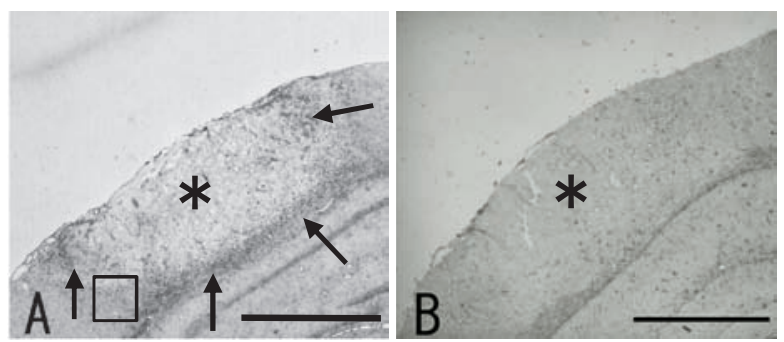

Fig. 1 In situ hybridization for OASIS mRNA in the cortical lesion at day 7 after cryo-injury. OASIS mRNA signals were visualized with the NBT/BCIP reaction using alkaline phosphatase. A: OASIS mRNA-positive cells were detected in the margin (arrows) of necrotic tissue (asterisk). B: Incubation with sense probe generated no detectable signals. Boxed area including the marginal zone was subjected to more detailed analysis in Figs. 2 and 3. Scale bars: $1 \mathrm{~mm}$.

with immunohistochemistry using various cell markers. Abundant GFAP-positive cells were detected in the region surrounding the necrotic core of the cortex at day 7 (Fig. 2B). They were classifiable into two groups: one including cells with a round shape and moderate GFAP immunoreactivity residing in or adjacent to the necrotic lesion (proximal reactive astrocytes, arrows in Fig. 2B), and another including cells with prominent processes and intense GFAPimmunoreactivity in the more distant region from the necrotic core (distal reactive astrocytes, arrowheads in Fig. 2B) (40). OASIS mRNA was detected primarily in proximal reactive astrocytes, but only scarcely in distal reactive astrocytes (Figs. 2A-C). When compared with the immunoreactivity for TAPA, a marker for proximal reactive astrocytes, cells expressing OASIS mRNA showed consistent TAPA-immunoreactivity (Figs. 2D-F). However, MAB328-immunoreacitive cells representing mature oligodendrocytes were only slightly overlapped with OASIS mRNA-positive cells (data not shown). Although oligodendrocytes were abundant in the surrounding region, they appeared to be devoid of OASIS mRNA. Similarly, the distribution of Iba1immunoreactive microglia differed greatly from that of OASIS mRNA-positive cells (Figs. 2G-I). We omitted the primary antibody reaction in the procedure and confirmed the absence of the positive reaction (data not shown). Collectively, these data suggest that OASIS mRNA was expressed primarily in proximal reactive astrocytes in a cryo-injured brain.

Double labeling in situ hybridization of OASIS and chondroitin sulfate proteoglycans

After brain injury, reactive astrocytes are known to express CSPGs, a set of substances that inhibit axo- nal growing and which thereby become a major impediment to the restoration of brain function $(6,11)$. To clarify the relation between OASIS and various CSPGs, double fluorescent labeling in situ hybridization was performed. At day 7 after cryo-injury, genes for CSPGs, including NG2 proteoglycan, versican, brevican, neurocan, and phosphacan were clearly induced to the similar extent in the gliotic area. Almost complete overlap of mRNA labeling was found for OASIS and all the examined CSPGs (Fig. 3). No significant signals were detected in adjacent sections hybridized with the sense probes (data not shown). These results suggest that induction of OASIS gene coincides with the expression of CSPG genes in reactive astrocytes.

\section{Inhibition of neurite outgrowth on OASIS-transfected cell membrane}

We next assessed neurite outgrowth of NG108-15 cells in the presence of cAMP on dishes coated with the membrane fraction derived from either vectortransfected or OASIS-transfected C6 cells. As noted, CSPGs produced by reactive astrocytes are a major impediment to the restoration of brain function (11), which is supported by the fact that growing axons of transplanted neurons stop at and turn away from the region rich in chondroitin sulfate moiety in spinal cord injury (6). As shown in Fig. 4, neurite outgrowth assay was conducted to determine whether OASIS contributes to the non-permissive environment for neurite outgrowth through the expression of CSPGs. Neurite outgrowth data (total neurite length per single cell; mean \pm SD) were $153.45 \pm$ $78.93 \mu \mathrm{m}$ on dishes coated with membrane fraction derived from vector-transfected cells (lane 1). However, outgrowth was much less on dishes coated with membrane fraction derived from stable C6 transfectants with OASIS (lanes 3 and 5; $83.95 \pm$ $49.56 \mu \mathrm{m}$ for OASIS 1 and $89.28 \pm 44.04 \mu \mathrm{m}$ for OASIS2, respectively, $P<0.0001$ ).

To examine whether the inhibitory effect of OASIS was exerted through the expression of CSPGs, the obtained membrane fractions were treated using chondroitinase $\mathrm{ABC}$ before use. Results revealed that neurite outgrowth on dishes coated with chondroitinase-treated membrane fraction was increased significantly (lanes 4 and 6; $164.54 \pm$ $85.46 \mu \mathrm{m}$ for OASIS1 and $150.36 \pm 77.64 \mu \mathrm{m}$ for OASIS2, respectively, $P<0.0001$ ); it was comparable to that on dishes coated with the membrane fraction derived from vector-transfected C6 cells (lane 1). No significant change was observed in the level of neurite outgrowth between dishes coated 

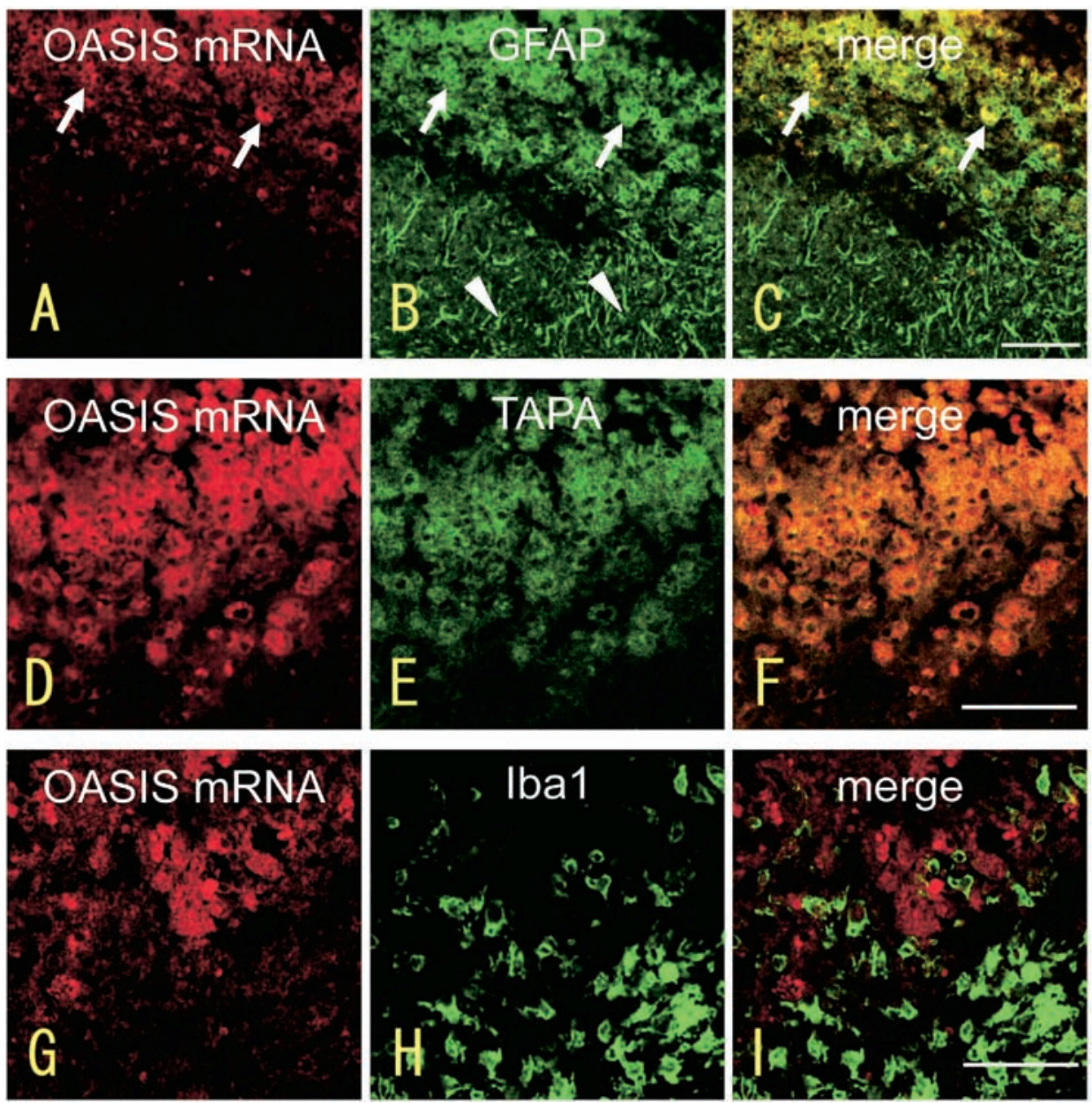

Fig. 2 Confocal images of in situ hybridization for OASIS mRNA combined with immunohistochemistry for various cell markers in adult mouse brain at day 7 after injury. Expression of OASIS mRNA (red, A, D, G) was examined using various marker antibodies (green), including GFAP (B), TAPA (E), and Iba1 (H). OASIS mRNA labeling (A) colocalizes with GFAPimmunoreactive, proximal reactive astrocytes ( $B$, arrows), but scarcely with distal reactive astrocytes (B, arrowheads). TAPAimmunoreactivity ( $E$, a marker for proximal reactive astrocytes) consistently coincides with OASIS mRNA labeling (D). On the other hand, OASIS mRNA labeling $(G)$ is hardly overlapped with Iba1-immunoreactive microglia $(H)$. Scale bars: $200 \mu \mathrm{m}$ $(A-C), 100 \mu \mathrm{m}(\mathrm{D}-\mathrm{I})$.

with untreated (lane $1 ; 153.45 \pm 78.93 \mu \mathrm{m}$ ) and chondroitinase-treated membrane fractions (lane 2; $159.34 \pm 79.15 \mu \mathrm{m})$ derived from vector-transfected cells. Collectively, the data suggest that OASIS contributes to the non-permissive environment for neurite outgrowth through the expression of CSPGs.

\section{DISCUSSION}

OASIS, a member of the CREB/ATF transcription factor family, was first identified as a gene expressed in long-term cultured mouse astrocytes (20). OASIS mRNA is expressed on day 3 to day 14 after brain injury, in the area surrounding necrotic tissue, with peak expression occurring on day 7; the cellular expression localization remains unclear (20). In this study, we first determined details of the cell types expressing OASIS mRNA by double-labeling with various cell markers.

Reactive astrocytes, the main scar component, are characterized by the intense expression of GFAP (8, 9 , 39). Based on their localization and the differential expression patterns of various molecules, reactive astrocytes can be grouped into two types: proximal reactive astrocytes, with a round and moderate GFAP immunoreactivity residing in or adjacent to the necrotic lesion, and distal reactive astrocytes, with prominent processes and intense GFAP immunoreactivity in the more distant region from the necrotic core (40). In the present study, we demon- 

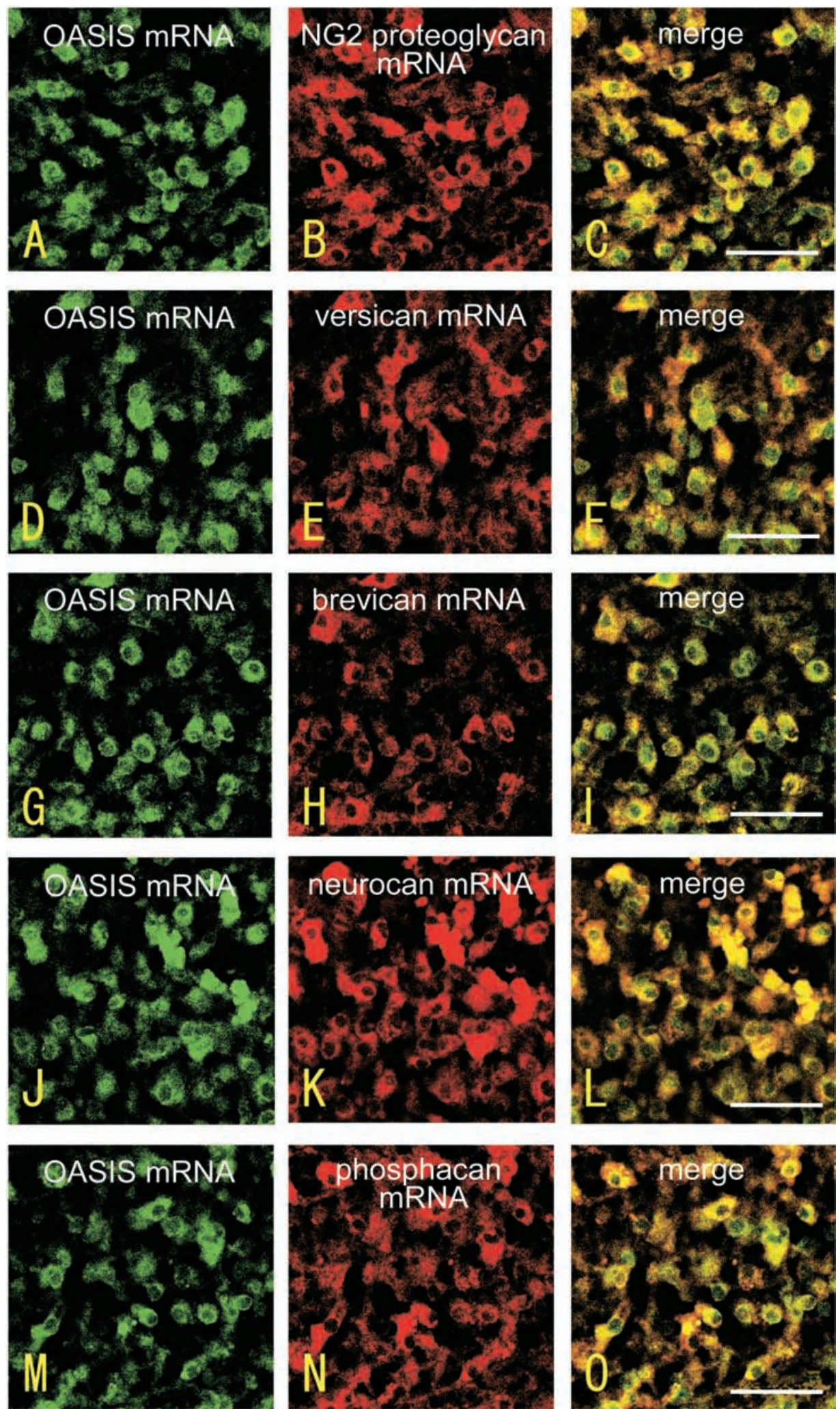

Fig. 3 Confocal images of double labeling in situ hybridization for OASIS mRNA and CSPG core protein mRNAs. OASIS mRNA-positive cells (green, A, D, G, J, M) almost completely coincide with cells labeled for CSPG core protein mRNAs (red), including NG2 proteoglycan $(B)$, versican $(E)$, brevican $(H)$ neurocan $(K)$ and phosphacan $(N)$ in injured brain tissue. Scale bars: $50 \mu \mathrm{m}$. 


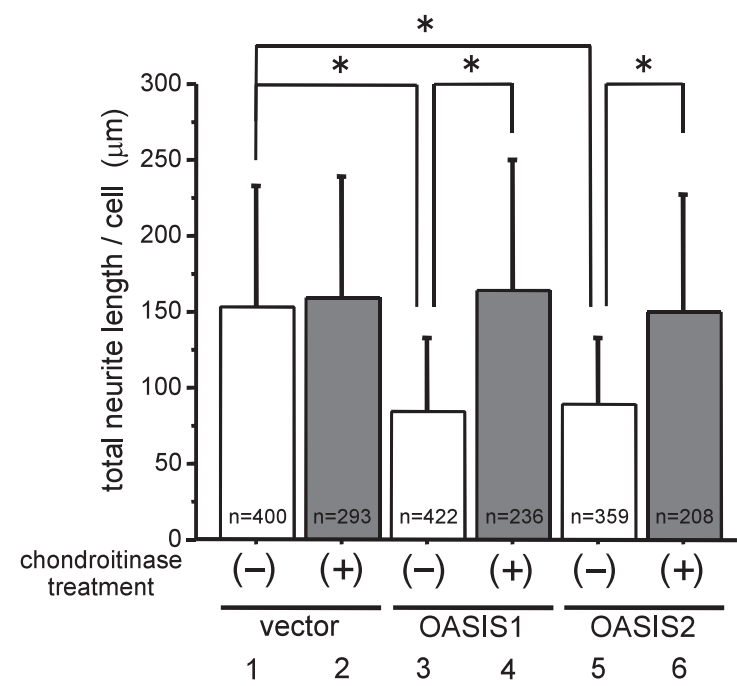

Fig. 4 Neurite outgrowth assay of NG108-15 cells. NG108-15 cells were cultured for 7 days with high-glucose DMEM in the presence of $10 \mathrm{mM}$ cAMP on dishes coated with membrane fraction of vector-transfected stable clone (lane 1) or OASIS-transfected stable clones (lane 3 for OASIS1 and lane 5 for OASIS2). Similar experiment was also performed on dishes coated with chondroitinase-treated membrane fraction of those cells (lanes 2, 4, 6). A total neurite length per single cell was measured for each condition. The number of neurites per cell ranged from 2 to 5 . The number of cells analyzed was depicted at the bottom of each column. The data were mean \pm SD and subjected to statistical analyses using ANOVA followed by Scheffé's test for post hoc multiple comparisons. ${ }^{*} P<0.0001$

strated that OASIS is detected primarily in proximal reactive astrocytes that are identified by TAPA/ CD81 (a member of the tetraspanin family), which is upregulated in proximal, but not in distal, reactive astrocytes of glial scar after CNS injury (4, 7, 37). No colocalization of OASIS mRNA with immunoreactivities for MAB328 (an oligodendrocyte marker) or Iba1 (a microglia marker) was observed. Therefore, OASIS is unlikely to be expressed in mature oligodendrocytes or macrophage/microglia.

Previous reports have described that gliotic tissue expresses CSPGs, which regulate cell migration, axonal growth, and neural patterning during development $(10,19,32)$. NG2 proteoglycan, versican, neurocan, and phosphacan are present at lower levels in mature brains of normal adults than in the immature brains of embryos (11). Brevican is also observed at low levels, except in the region of the ventricular zone, where the generation and initial migration of glial cells occur during development (27), but diffusely increases in both neurons and glial cells in the adult brain (43). Regarding brain injury, the astroglial scar inhibits axonal regeneration, which is mostly attributed to CSPGs secreted from reactive astrocytes. For example, brevican, phosphacan, and neurocan mRNAs are upregulated on GFAP-positive reactive astrocytes in the CNS glial scar $(17,33,44)$. Furthermore, NG2 proteoglycan, a well-known marker of oligodendrocyte precursor cells, is induced in reactive astrocytes and macrophages after CNS injury (11, 29, 31). In this study, we show clearly that induction of OASIS gene coincides with the expression of CSPG genes in reactive astrocytes.

In terms of the functional roles of CSPGs, NG2 proteoglycan is a major inhibitory proteoglycan to axonal growth in vitro (12). It loses its inhibitory functions when glycosaminoglycan chains are removed. Major versican isoform V2 strongly inhibits neurite outgrowth of the central and peripheral neurons (41). Brevican inhibits neurite outgrowth and the attachment of cerebellar granule neurons to the coated surface (46). Phosphacan exerts both a neurite outgrowth-promoting function for the embryonic hippocampal neurons and an inhibitory function for the sensory neurons $(5,14)$. In the other experimental paradigm, phosphacan, which is upregulated by TGF- $\beta$ in the brain lesion, inhibits axonal growth (42).

Previous study shows that OASIS interacts with CRE consensus sequence (36), which is found in some of the CSPG core protein genes (34). In addition, recent study reports a microarray expression profiling in cells expressing active OASIS and an increase in CSPG expression in these cells (45). In the present study, we confirm that the expression of OASIS contributes to the non-permissive environment for neurite outgrowth of NG108-15 cells. The non-permissive environment elaborated by the membrane derived from OASIS-transfected C6 cells reverted to a permissive state through treatment of chondroitinase, suggesting that CSPGs are responsible for the effect, which is consistent with descriptions in earlier reports described above.

After brain injury, however, other PGs such as heparan sulfate proteoglycans (HSPGs), including syndecan (22), glypican-1 (18), and testican-1 (23), have also been shown to be expressed in the region surrounding necrotic tissue. Previous study suggests that CSPGs inhibit neurite outgrowth in vitro (13) whereas HSPGs have high affinity for growth factors and heparin-binding growth molecules, including FGF-2 and pleiotrophin, which suggests permissive role of HSPGs for regenerating axons (47). Further study is warranted to examine whether or not OASIS and HSPG core proteins are coex- 
pressed in reactive astrocytes after brain injury. In addition, recent study shows that Egr-1, the early growth response 1 transcription factor, is also expressed in reactive astrocytes and regulates the transcription of CSPG protein genes (3). These reports suggest that the expression of extracellular matrix after brain injury is regulated by various mechanisms, which might depend on the time course after injury and the environmental conditions.

In summary, we showed that OASIS mRNA is upregulated in proximal reactive astrocytes of the injured brain. OASIS triggers proteoglycan production in scar tissue, which creates a non-permissive environment for regenerating axons. The present findings provide clues to devise regenerative gene therapies for brain injury and to investigate the mechanisms by which adult neurons fail to regenerate efficiently.

\section{Acknowledgments}

This work was supported by a Grant-in-Aid from the Ministry of Education, Culture, Sports, Science, and Technology (MEXT) of Japan (K.I.).

\section{REFERENCES}

1. Akita K, Toda M, Hosoki Y, Inoue M, Fushiki S, Oohira A, Okayama M, Yamashina I and Nakada H (2004) Heparan sulphate proteoglycans interact with neurocan and promote neurite outgrowth from cerebellar granule cells. Biochem $J$ 383, 129-138.

2. Asher RA, Morgenstern DA, Shearer MC, Adcock KH, Pesheva P and Fawcett JW (2002) Versican is upregulated in CNS injury and is a product of oligodendrocyte lineage cells. J Neurosci 22, 2225-2236.

3. Beck H, Semisch M, Culmsee C, Plesnila N and Hatzopoulos AK (2008) Egr-1 regulates expression of the glial scar component phosphacan in astrocytes after experimental stroke. Am J Pathol 173, 77-92.

4. Clarke K and Geisert EE, Jr. (1998) The target of the antiproliferative antibody (TAPA) in the normal and injured rat retina. Mol Vis 4, 3.

5. Clement AM, Nadanaka S, Masayama K, Mandl C, Sugahara K and Faissner A (1998) The DSD-1 carbohydrate epitope depends on sulfation, correlates with chondroitin sulfate D motifs, and is sufficient to promote neurite outgrowth. J Biol Chem 273, 28444-28453.

6. Davies SJ, Goucher DR, Doller C and Silver J (1999) Robust regeneration of adult sensory axons in degenerating white matter of the adult rat spinal cord. J Neurosci 19, 5810-5822.

7. Dijkstra S, Geisert EJ, Gispen WH, Bar PR and Joosten EA (2000) Up-regulation of CD81 (target of the antiproliferative antibody; TAPA) by reactive microglia and astrocytes after spinal cord injury in the rat. J Comp Neurol 428, 266-277.

8. Eng LF and Ghirnikar RS (1994) GFAP and astrogliosis. Brain Pathol 4, 229-237.

9. Eng LF, Ghirnikar RS and Lee YL (2000) Glial fibrillary acidic protein: GFAP-thirty-one years (1969-2000). Neurochem Res 25, 1439-1451.

10. Faissner A and Steindler D (1995) Boundaries and inhibitory molecules in developing neural tissues. Glia 13, 233-254.

11. Fawcett JW and Asher RA (1999) The glial scar and central nervous system repair. Brain Res Bull 49, 377-391.

12. Fidler PS, Schuette K, Asher RA, Dobbertin A, Thornton SR, Calle-Patino Y, Muir E, Levine JM, Geller HM, Rogers JH, Faissner A and Fawcett JW (1999) Comparing astrocytic cell lines that are inhibitory or permissive for axon growth: the major axon-inhibitory proteoglycan is NG2. J Neurosci 19, 8778-8788.

13. Galtrey CM and Fawcett JW (2007) The role of chondroitin sulfate proteoglycans in regeneration and plasticity in the central nervous system. Brain Res Rev 54, 1-18.

14. Garwood J, Schnadelbach O, Clement A, Schutte K, Bach A and Faissner A (1999) DSD-1-proteoglycan is the mouse homolog of phosphacan and displays opposing effects on neurite outgrowth dependent on neuronal lineage. J Neurosci 19, 3888-3899.

15. Geisert EE, Jr., Yang L and Irwin MH (1996) Astrocyte growth, reactivity, and the target of the antiproliferative antibody, TAPA. J Neurosci 16, 5478-5487.

16. Graeber MB, Lopez-Redondo F, Ikoma E, Ishikawa M, Imai Y, Nakajima K, Kreutzberg GW and Kohsaka S (1998) The microglia/macrophage response in the neonatal rat facial nucleus following axotomy. Brain Res 813, 241-253.

17. Haas CA, Rauch U, Thon N, Merten T and Deller T (1999) Entorhinal cortex lesion in adult rats induces the expression of the neuronal chondroitin sulfate proteoglycan neurocan in reactive astrocytes. J Neurosci 19, 9953-9963.

18. Hagino S, Iseki K, Mori T, Zhang Y, Sakai N, Yokoya S, Hikake T, Kikuchi S and Wanaka A (2003) Expression pattern of glypican-1 mRNA after brain injury in mice. Neurosci Lett 349, 29-32.

19. Herndon ME and Lander AD (1990) A diverse set of developmentally regulated proteoglycans is expressed in the rat central nervous system. Neuron 4, 949-961.

20. Honma Y, Kanazawa K, Mori T, Tanno Y, Tojo M, Kiyosawa H, Takeda J, Nikaido T, Tsukamoto T, Yokoya S and Wanaka A (1999) Identification of a novel gene, OASIS, which encodes for a putative CREB/ATF family transcription factor in the long-term cultured astrocytes and gliotic tissue. Brain Res Mol Brain Res 69, 93-103.

21. Imai Y, Ibata I, Ito D, Ohsawa K and Kohsaka S (1996) A novel gene iba1 in the major histocompatibility complex class III region encoding an EF hand protein expressed in a monocytic lineage. Biochem Biophys Res Commun 224, 855-862.

22. Iseki K, Hagino S, Mori T, Zhang Y, Yokoya S, Takaki H, Tase C, Murakawa M and Wanaka A (2002) Increased syndecan expression by pleiotrophin and FGF receptor-expressing astrocytes in injured brain tissue. Glia 39, 1-9.

23. Iseki $\mathrm{K}$, Hagino $\mathrm{S}$, Zhang $\mathrm{Y}$, Mori $\mathrm{T}$, Sato N, Yokoya S, Hozumi Y, Goto K and Tase C (2011) Altered expression pattern of testican-1 mRNA after brain injury. Biomed Res 32, 373-378.

24. Ishikawa M, Tsukamoto $\mathrm{T}$ and Yamamoto $\mathrm{T}$ (1996) Longterm cultured astrocytes inhibit myelin formation, but not axonal growth in the co-cultured nerve tissue. Mult Scler 2, 91-95.

25. Ito D, Imai Y, Ohsawa K, Nakajima K, Fukuuchi Y and Kohsaka S (1998) Microglia-specific localisation of a novel calcium binding protein, Iba1. Brain Res Mol Brain Res 57, $1-9$. 
26. Ito D, Tanaka K, Suzuki S, Dembo T and Fukuuchi Y (2001) Enhanced expression of Iba1, ionized calcium-binding adapter molecule 1, after transient focal cerebral ischemia in rat brain. Stroke 32, 1208-1215.

27. Jaworski DM, Kelly GM and Hockfield S (1995) The CNSspecific hyaluronan-binding protein BEHAB is expressed in ventricular zones coincident with gliogenesis. $J$ Neurosci 15, 1352-1362.

28. Jaworski DM, Kelly GM and Hockfield S (1999) Intracranial injury acutely induces the expression of the secreted isoform of the CNS-specific hyaluronan-binding protein BEHAB/ brevican. Exp Neurol 157, 327-337.

29. Jones LL, Yamaguchi Y, Stallcup WB and Tuszynski MH (2002) NG2 is a major chondroitin sulfate proteoglycan produced after spinal cord injury and is expressed by macrophages and oligodendrocyte progenitors. J Neurosci 22, 2792-2803.

30. Kondo S, Murakami T, Tatsumi K, Ogata M, Kanemoto S, Otori K, Iseki K, Wanaka A and Imaizumi K (2005) OASIS, a CREB/ATF-family member, modulates UPR signalling in astrocytes. Nat Cell Biol 7, 186-194.

31. Levine JM (1994) Increased expression of the NG2 chondroitin-sulfate proteoglycan after brain injury. $J$ Neurosci 14, 4716-4730.

32. Margolis RU and Margolis RK (1997) Chondroitin sulfate proteoglycans as mediators of axon growth and pathfinding. Cell Tissue Res 290, 343-348.

33. McKeon RJ, Jurynec MJ and Buck CR (1999) The chondroitin sulfate proteoglycans neurocan and phosphacan are expressed by reactive astrocytes in the chronic CNS glial scar. J Neurosci 19, 10778-10788.

34. Naso MF, Zimmermann DR and Iozzo RV (1994) Characterization of the complete genomic structure of the human versican gene and functional analysis of its promoter. $J$ Biol Chem 269, 32999-33008.

35. Nikaido T, Yokoya S, Mori T, Hagino S, Iseki K, Zhang Y, Takeuchi M, Takaki H, Kikuchi S and Wanaka A (2001) Expression of the novel transcription factor OASIS, which belongs to the CREB/ATF family, in mouse embryo with special reference to bone development. Histochem Cell Biol 116, 141-148.

36. Nikaido T, Iseki K, Mori T, Takaki H, Yokoya S, Hagino S, Takeda J, Zhang Y, Takeuchi M, Kikuchi S and Wanaka A (2002) Expression of OASIS, a CREB/ATF family transcription factor, in CNS lesion and its transcriptional activity.
Brain Res Mol Brain Res 108, 129-138.

37. Peduzzi JD, Grayson TB, Fischer FR and Geisert EE, Jr. (1999) The expression of TAPA (CD81) correlates with the reactive response of astrocytes in the developing rat CNS. Exp Neurol 160, 460-468.

38. Perides G, Asher RA, Lark MW, Lane WS, Robinson RA and Bignami A (1995) Glial hyaluronate-binding protein: a product of metalloproteinase digestion of versican? Biochem $J$ 312, 377-384.

39. Petito CK, Morgello S, Felix JC and Lesser ML (1990) The two patterns of reactive astrocytosis in postischemic rat brain. J Cereb Blood Flow Metab 10, 850-859.

40. Ridet JL, Malhotra SK, Privat A and Gage FH (1997) Reactive astrocytes: cellular and molecular cues to biological function. Trends Neurosci 20, 570-577.

41. Schmalfeldt M, Bandtlow CE, Dours-Zimmermann MT, Winterhalter KH and Zimmermann DR (2000) Brain derived versican V2 is a potent inhibitor of axonal growth. $J$ Cell Sci 113, 807-816.

42. Schnadelbach O, Mandl C and Faissner A (1998) Expression of DSD-1-PG in primary neural and glial-derived cell line cultures, upregulation by TGF- $\beta$, and implications for cellsubstrate interactions of the glial cell line Oli-neu. Glia 23, 99-119.

43. Seidenbecher CI, Gundelfinger ED, Bockers TM, Trotter J and Kreutz MR (1998) Transcripts for secreted and GPIanchored brevican are differentially distributed in rat brain. Eur J Neurosci 10, 1621-1630.

44. Thon N, Haas CA, Rauch U, Merten T, Fassler R, Frotscher M and Deller T (2000) The chondroitin sulphate proteoglycan brevican is upregulated by astrocytes after entorhinal cortex lesions in adult rats. Eur J Neurosci 12, 2547-2558.

45. Vellanki RN, Zhang L, Guney MA, Rocheleau JV, Gannon $\mathrm{M}$ and Volchuk A (2010) OASIS/CREB3L1 induces expression of genes involved in extracellular matrix production but not classical endoplasmic reticulum stress response genes in pancreatic beta-cells. Endocrinology 151, 4146-4157.

46. Yamada H, Fredette B, Shitara K, Hagihara K, Miura R, Ranscht B, Stallcup WB and Yamaguchi Y (1997) The brain chondroitin sulfate proteoglycan brevican associates with astrocytes ensheathing cerebellar glomeruli and inhibits neurite outgrowth from granule neurons. J Neurosci 17, 7784-7795.

47. Yamaguchi Y (2001) Heparan sulfate proteoglycans in the nervous system: their diverse roles in neurogenesis, axon guidance, and synaptogenesis. Semin Cell Dev Biol 12, 99-106. 